\title{
EfFECTS OF Muscle FATIgUe ON THE USABILITY OF A MYOELECTRIC HUMAN-COMPUTER INTERFACE
}

\author{
Alexander G. Barszap* \\ Ida-Maria Skavhaug* \\ Sanjay S. Joshi** \\ *These authors contributed equally to the paper \\ **Corresponding author: maejoshi@ucdavis.edu
}

All authors are with the University of California, Davis, Department of Mechanical and Aerospace Engineering, One Shields Avenue Davis, CA 95616 


\section{ABSTRACT}

Electromyography-based human-computer interface development is an active field of research. However, knowledge on the effects of muscle fatigue on specific devices is limited. We have developed a novel myoelectric human-computer interface in which subjects continuously navigate a cursor to targets by manipulating a single surface electromyography (sEMG) signal. Two-dimensional control is achieved through simultaneous adjustments of power in two frequency bands through a series of dynamic low-level muscle contractions. Here, we investigate the potential effects of muscle fatigue on the use of our interface. In the first session, eight subjects completed 300 cursor-to-target trials without breaks; four using a wrist muscle and four using a head muscle. The wrist subjects returned for a second session in which a static fatiguing exercise took place at regular intervals in-between cursor-to-target trials. In the first session we observed no declines in performance as a function of use, even after the long period of use. In the second session, we observed clear changes in cursor trajectories, paired with a target-specific decrease in hit rates.

Keywords: Surface Electromyography; sEMG; Myoelectric Interface; Cursor Control; Median Frequency Shift; Muscle Fatigue; Human-Computer Interface 


\section{INTRODUCTION}

Several electromyography (EMG)-based devices for control of computers and external machines have recently been reported in the literature, which all have the collective aim of assisting individuals with varying degrees of physical disability (Chin, Barreto, Cremades, \& Adjouadi, 2008; Cler \& Stepp, 2015; Moon, Lee, Chu, \& Mun, 2005; Oskoei \& Hu, 2007; Song, Jung, Lee, \& Bien, 2009). Common concerns for any EMG interface are fatigue-related failures and/or loss of performance; however research on the effects of muscle fatigue on EMG-controlled devices has been relatively limited. It is important to understand the potential consequences of fatigue in interface usability, so that fatigue can be monitored and compensated for (if required). Here, we add to the limited body of work by examining the effects of fatigue on a novel single-site myoelectric human-computer interface $(\mathrm{HCl})$ controlled via contractions of a head muscle (the auricularis posterior; AP) and a wrist muscle (the Extensor Pollicis Longus; EPL). We monitored interface use during a canonical cursor-to-target task (Perez-Maldonado, Wexler, \& Joshi, 2010; Skavhaug, Lyons, Nemchuk, Muroff, \& Joshi, 2016, Wolpaw and McFarland 2004) across two sessions. In the first session, we asked subjects to complete 300 trials without breaks (2.5-3 hours of continuous use). In the second session, we asked subjects to intermittently complete deliberate fatiguing exercises (EPL subjects only) between each block of 30 trials (again totaling 300 cursor-to-target trials during 2.5-3 hour long time interval).

\subsection{Novel Single Site MyoeleCtRic INTERfaCE}

We employ a previously described novel myoelectric $\mathrm{HCl}$, which requires subjects to manipulate the power spectrum in a single surface EMG (sEMG) signal through low-level dynamic muscle contractions (Perez-Maldonado et al., 2010; Vernon \& Joshi, 2011, Skavhaug, Bobell, Vernon, \& Joshi, 2012; Lyons \& Joshi, 2013; Skavhaug, Lyons, Nemchuk, Muroff, \& Joshi, 2016). The ultimate purpose of the sEMGbased device is the translation to a practical user interface for people with paralysis. Although it may seem paradoxical to base a $\mathrm{HCl}$ for people with paralysis on muscle contractions, the majority of even the most severely paralyzed have access to some spared muscles. For example, individuals with high spinal cord injuries usually have access to muscles that are innervated the brainstem (i.e. muscles on the head). Muscles innervated at the spinal cord may be viable alternatives for patients suffering certain medical conditions, in which limited access to some muscles are spared (such as spinal muscular atrophy; SMA). The idea of using spared muscle abilities for interface control is not a novel concept. For example, Cler and Stepp (2015) report a sEMG-based keyboard system for spelling, which involved the recording from five facial muscle sites. SEMG has also been used in cursor control paradigms, where positions were determined by EMG recorded on the neck and/or the face (Hands \& Stepp, 2016; Thorp, Larson, \& Stepp, 2014; Williams \& Kirsch, 2008). Other common applications of such sEMG-based interfaces are wheelchair control and prosthetics control (Choi, Sato, \& Koike, 2006; Felzer \& Freisleben, 2002; Moon, et al., 2005; Nguyen \& Nguyen, 2011; Oonishi, Oh, \& Hori, 2008; Roche, et al., 2014; Wei, Hu, \& Yuan, 2009; Zecca et al., 2002). 
The current study addresses sEMG-based devices used for computer control, such as moving a cursor on a computer screen. When a user with a disability achieves control of a mouse cursor, this may open up many possibilities for manipulating his or her environment. For example, Vernon \& Joshi (2011) reported a case study with an advanced SMA patient, who contracted an auricularis muscle to navigate a cursor to one of many targets on a cell phone screen. He selected a target by first moving the cursor to the desired target (command) and then relaxed the muscle to return the cursor to a rest area on the screen to confirm the selection. Hence, the return of the cursor (via muscle relaxation) represented the traditional 'mouse click'. In a more recent study, a subject with C3-C4 spinal cord injury remotely controlled a tele-presence robot by sending commands (and receiving video feedback) via Wi-Fi using a tablet-based version of the $\mathrm{HCl}$ (Lyons \& Joshi, 2013).

sEMG-based cursor-to-target paradigms typically includes one recording site per movement direction (Cler \& Stepp, 2015; Williams \& Kirsch, 2008). The most novel aspect of our interface is that we extract two control channels from one single signal. The design was inspired by the desire to minimize the number of electrodes needed for two-dimensional control. A minimal recording configuration is preferable for both cosmetic reasons and because some users simply do not have two corresponding muscles sites available. This was the case with one of our previous subjects with advanced SMA, who was confined to lying on one side of his head (see Vernon \& Joshi, 2011). Users achieve two-dimensional control from one signal by actively placing specified amounts of power in two separate frequency bands. The power in these bands are placed through a linear transformation to achieve position in twodimensional space (as will be described in detail in Method Section 2.2). In this sense, cursor position is proportional to the sEMG power in each band.

Our initial studies were inspired by the original work of Basmajian (1963), which showed that subjects could voluntarily control the firing rates of individual motor units, and a key brain-computer interface study by Wolpaw and McFarland (2004), which showed that subjects could voluntarily adjust the power profile of their EEG signals in two frequency bands. Based on these studies, we hypothesized that subjects may be able to modulate the firing rates of multiple motor units to create power profiles that can be translated to cursor positions on the screen. Proof of concept and user studies for this interface have been described in a series of previous articles, reporting results from experiments using both the AS muscle site, located above the ear, and the EPL muscle site on the wrist (Perez-Maldonado, et al., 2010; Skavhaug, Bobell, Vernon, \& Joshi, 2012; Lyons \& Joshi, 2013; Skavhaug, et al., 2016). Most recently, Skavhaug et al. (2016) described the learning curve stretching across eight sessions of interface use (approximately 45 minutes each) for a group of 12 naïve subjects. This study showed that cursor-totarget performance increased significantly from an initial $48 \%$ to $71 \%$ at the end of the last session (target sizes equaled $1 \%$ of total screen size), and that subjects were achieving greater proficiency over cursor paths after practice. It is possible that subjects may have continued to improve past the $71 \%$ success rate observed at session 8 , however most of the learning seemed to take place during the first three sessions of practice, after which the learning curve plateaued. This study also indicated that much 
higher success rates may be achieved using larger targets. Based on these observations, we estimate that for users to become relatively skilled at controlling the $\mathrm{HCl}$, they require 2-3 hours of practice.

Although subjects improve interface proficiency with practice, it is unlikely that they fully understand how the new motor skill is accomplished, as it involves esoteric notions of signal power. For this reason, naïve subjects are instructed to approach the cursor control task through a trial-and-error strategy, in which they contract the muscle in different ways to attempt to hit targets on the screen. With practice subjects appear to develop an understanding of the relationship between contractions and cursor movements (as reflected in increased target hitting rates and decreased time-to-target; see Skavhaug et al., 2016), although this understanding may not be easily articulated. The creation of power profiles involves the tuning of multiple motor units that coordinate the contraction of the muscle from which the signal is recorded. To vary the amount of relative power in each frequency band, subjects must modulate the number of active units and their firing rates, however the precise way in which this is achieved is yet to be described (this will likely require intramuscular recordings). In terms of the nature of the motor skill that subjects learn, we believe there are two possibilities: either the subjects are learning a one-to-one mapping of specific contractions and associated target locations on the screen, or, they learn to continuously modulate the power bands to move the cursor to any position on the screen (see discussion in Skavhaug et al. 2016). Finally, it is important to note that we do not preclude the possibility of contributions by one or several neighboring muscles to the single site SEMG signal (we emphasize that our device represents a single site, rather than single muscle interface); although we believe it is dominated by the muscle the sensor is placed upon. The degree of co-contraction by additional muscles is likely a function of the relative isolation of that muscle, hence it is possible that the signal recorded at the EPL is 'less pure' than the signal recorded at the auricularis muscles on the head.

\subsection{Muscle fatigue}

It is well known that muscle fatigue changes the recruitment of motor units contributing to a muscle contraction, which in turn changes the nature of any sEMG signal measured at that muscle (GonzalezIzal, Malanda, Gorostiaga, \& Izquierdo, 2012). An important consideration relating to any EMG-driven device is therefore the potential effects of muscle fatigue on usability. In muscle physiology, it has been proposed that sustained static isometric contractions may cause an increase in EMG signal amplitude along with a compression of the frequency spectrum (Gonzalez-Izal et al., 2012; Mannion, Connolly, Wood, \& Dolan, 1997; Merletti, Knaflitz, \& DeLuca, 1992). For example, for proportional-control based systems (such as those in which the EMG signal is rectified and low-pass filtered to produce an output proportional to the total power in the signal), Park and Meek (1993) proposed a fatigue compensator preprocessor to reverse the effects of muscle fatigue on the frequency spectrum of an EMG signal. Song, et al. (2009) found that pattern recognition based systems, such as those that rely on classification from a variety of EMG channels, are especially susceptible to the effects of fatigue and proposed a fatigue compensation method for EMG-based pattern classification. As our method uses low levels of dynamic contractions at a single sEMG site to achieve multidimensional continuous control, this technique is very 
different from classification control techniques, (e.g. Jiang et al., 2013; Young et al., 2014) and other common signal processing techniques, which require at least one muscle site per degree-of-freedom for continuous control (e.g. Engelhart \& Hudgins, 2003; Scheme \& Engelhard, 2011). It is therefore unclear from the literature how fatigue will affect the usability of our $\mathrm{HCl}$.

In the current study, eight subjects used our interface for an extended period of time, some of whom took part in static external muscle exercises to accelerate the rate of fatigue. In each session, subjects performed 300 consecutive cursor-to-target trials. The approximate session completion time was 2.5-3 hours, including setup, calibration, and testing ${ }^{1}$. Subjects momentarily relaxed their muscle after each trial to start a new trial. Although it is possible that a full day's use of the interface would amount to a higher number of total trials, it is unlikely that any person would be continuously using the $\mathrm{HCl}$ without any chance of rest. Previous experiments involving use of the device showed that completion of full tasks took less than ten minutes of use. This was the case in both Lyons and Joshi (2013), where a spinal cord injured subject controlled a wheeled robot to navigate an obstacle course, and in Weisz, Barszap, Joshi, \& Allen (2014), where the same subject controlled a robotic arm in a remote location to identify, approach, and lift a bottle. For tasks similar to these, it is very unlikely that the system would be used for hours without a break.

We were interested in any consequences of fatigue on cursor-to-target performance and the general ability to retain control of the movements of the cursor throughout the experiment. In particular, we measured target-hitting rates (success rates) and the time it took the subject to reach the target in seconds (time-to-target) across the 300 trials. In addition, we observed the cursor's position on the screen to monitor any changes in cursor movements as a function of use. We expected that fatigue, if present, would cause a decline in overall performance and ability to control the cursor's trajectories.

\section{METHODS}

\subsection{SUBJECTS}

Eight subjects participated in this study (seven able-bodied subjects and one subject with paralysis). Each of the subjects had previously participated in myoelectric control experiments within our laboratory, including studies of other devices with similar control strategies. However, none of the subjects had used the specific computer-based hardware of the current experiment. We chose subjects with prior exposure to minimize early learning effects as a confounding factor (Skavhaug et al., 2016) and to ensure limited familiarity with the procedure to reduce the time needed for pre-experimental briefing/introduction. Subjects were not chosen based on a performance criterion. Table 1 lists all 
subjects and pertinent information. Participants were fully consented according to the UC Davis IRB protocol (\#251192) and compensated with a \$20 gift card per session for participating in the study.

Table 1. Subject information. *Individual with incomplete C3-C4 spinal cord injury (limited upper limb mobility).

\begin{tabular}{|c|c|c|c|c|c|}
\hline Subject & Age & Gender & Muscle & $\begin{array}{c}\text { Previous } \\
\text { Training } \\
\text { (hours) }\end{array}$ & $\begin{array}{c}\text { Time since last } \\
\text { HCl study }\end{array}$ \\
\hline 1 & 29 & M & EPL & 2 & 24 months \\
\hline 2 & 23 & M & EPL & 4 & 5 months \\
\hline 3 & 19 & M & EPL & 4 & 17 months \\
\hline 4 & 19 & F & EPL & 4 & 10 months \\
\hline 5 & 21 & M & AP & 6 & 2 days \\
\hline 6 m & 30 & M & AP & 8 & 12 months \\
\hline 7 & 20 & F & AP & 4 & 3 months \\
\hline 8 & 20 & F & AP & 4 & 6 months \\
\hline
\end{tabular}

\subsection{Procedure}

One differential SEMG signal was collected from the extensor pollicis longus (EPL) muscle site for subjects 1-4 and from the auricularis posterior (AP) muscle site for subjects 5-8 (see Figure $1 a$ and $b$ ). Previous work by our laboratory has shown that both of these muscles can be used as inputs to the sEMG system (Lyons \& Joshi, 2013; Perez-Maldonado, et al., 2010; Skavhaug et al., 2016). The AP muscle is used to move the ear back and forth, and serves as a good myoelectric interface candidate for those with high spinal cord injury. The EPL muscle is used for extending the thumb, and was also chosen in this experiment to allow us to induce fatigue with a simple external static exercise as shown in Figure 1c. The EPL is a useful muscle for interface testing, as healthy subjects are familiar with flexing their thumb, and therefore require no additional training to access the muscle. The AP muscle was not externally fatigued in this study.

For recordings from the EPL muscle (subjects 1-4), a set of disposable $\mathrm{Ag}-\mathrm{AgCl}$ snap electrodes (6.3 mm diameter) were placed on the base of the subject's dominant hand along the axis of the muscle with an approximate $1.5 \mathrm{~cm}$ inter-electrodes distance. A reference electrode was placed on the elbow. For recordings from the AP muscle, a set of non-disposable $\mathrm{Ag}-\mathrm{AgCl}$ cup electrodes (4 $\mathrm{mm}$ diameter) were placed behind the subject's dominant ear along the mastoid bone with an inter-electrode distance of approximately $1 \mathrm{~cm}$. A reference electrode was placed on ear lobe. The skin was cleansed with an alcohol wipe prior to applying the electrodes. The AP electrodes were the EL254S type from Biopac Systems Inc., and Ten20 conductive paste was used to hold the electrodes in place. The EPL electrodes 
were of the type ConMed 1620 Pediatric Huggables Electrodes held in place with Clear NeoDerm tape. Both kinds of electrodes were connected to a model Y03 preamplifier (www.motion-labs.com) with input impedance greater than $10^{8} \Omega, 15-2000 \mathrm{~Hz}$ signal bandwidth and gain of 300 . sEMG recordings can contain contributions from a variety of underlying muscles; however we assume that each of the muscle sites we selected has a dominant muscle contribution (EPL or AP).

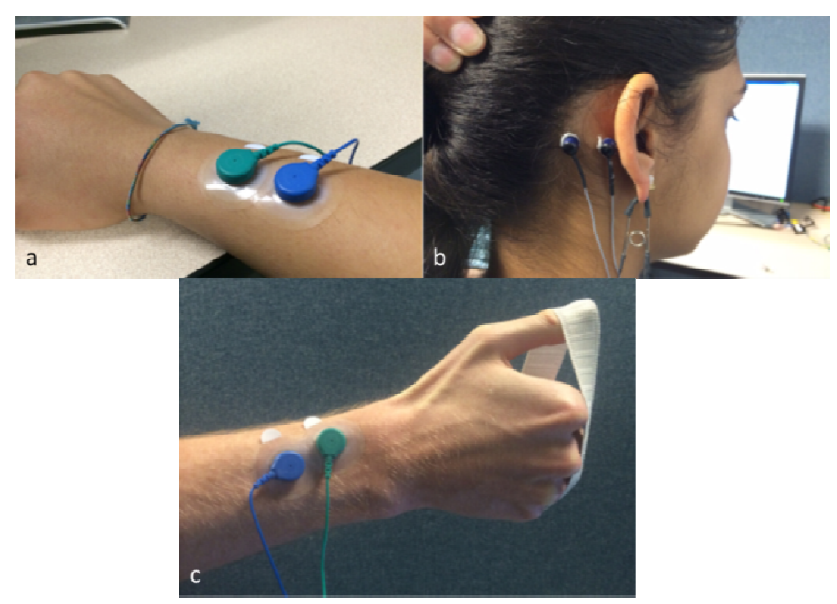

Figure 1. a) Sensors attached to the wrist to measure EPL muscle activity. b) Sensors attached behind ear to measure AP muscle activity. c) EPL subject performing fatiguing exercise.

The preamplifier measured a single analog differential sEMG signal that was fed into the microphone jack of a standard Dell desktop computer. Matlab ${ }^{\mathrm{TM}}$ r2011b (32 bit version) was used to read in the signal through the analog to digital converter (ADC) in the computer's sound card. The sEMG signal was sampled at $8000 \mathrm{~Hz}$ and then down sampled to $4000 \mathrm{~Hz}$ and sent through a series of 4th order Butterworth notch filters as described in Mello, Oliveira, and Nadal (2007) to filter out $60 \mathrm{~Hz}$ noise and its harmonics. The single sEMG signal was then converted into two simultaneous power band signals by duplicating the signal and passing each through a separate 4th order Butterworth IIR band pass filter created with the Matlab Signal Processing Toolbox. The first signal (Band 1) was band pass filtered between $80-100 \mathrm{~Hz}$. The second signal (Band 2) was band pass filtered between 130-150 Hz. These bands were selected based on visual inspection of several maximum voluntary contractions (MVC) from previous pilot subjects. We chose two non-overlapping bands of the same width which appeared to produce comparable powers during contractions. We note that the value of personalized selection of bands is a separate question being studied in our lab (Perez-Maldonado, et al., 2010), but it is clear that personalized selection of bands is not necessary for use of the device (see Skavhaug, et al., 2016). The total power in each of the two resulting signals, (PowerB_1 and PowerB_2) in Equation 1, were computed using 1000 samples (0.25 seconds of data) per sample point by computing a FFT. The maximum partial power for each band was computed (MPB_1 and MPB_2 in Equation 1, see calibration below) and used to normalize the power signals. These two normalized powers (PowerB_1(t)/MPB_1 and PowerB_2(t)/MPB_2) were then linearly combined as shown in Equation 1 to calculate an 
instantaneous cursor position, represented as a percentage of the screen in each direction (value from 0-100).

Equation 1.

$$
\left[\begin{array}{l}
X(t) \\
Y(t)
\end{array}\right]=\left[\begin{array}{cc}
\frac{1}{E_{1}} & 0 \\
0 & \frac{1}{E_{2}}
\end{array}\right]\left[\begin{array}{cc}
1.75 & -0.75 \\
-0.75 & 1.75
\end{array}\right]\left[\begin{array}{l}
\frac{\text { Power }_{1}(t)}{M P B_{1}} \\
\frac{\operatorname{PowerB}_{2}(t)}{M P B_{2}}
\end{array}\right]
$$

The instantaneous cursor position ( $X$ and $Y$, a value between zero and 100 representing the cursor's position in each direction as a percent of the total screen dimension) was passed through a $5^{\text {th }}$ order linear-phase FIR filter using the Parks-McClellan algorithm (Matlab signal processing toolbox), with a cutoff frequency of $0.5 \mathrm{~Hz}$ in order to smooth the cursor movement. This produced an updated cursor position every 0.25 seconds $(4 \mathrm{~Hz})$. In order to present a visually smooth refresh rate to the screen, seven points were interpolated in a straight line between the last two calculated positions, increasing the cursor position refresh rate to $32 \mathrm{~Hz}$ and introducing a 0.25 second delay between the calculated cursor position and the displayed screen position. A flow chart of the entire signal processing pipeline is presented in Figure 2.
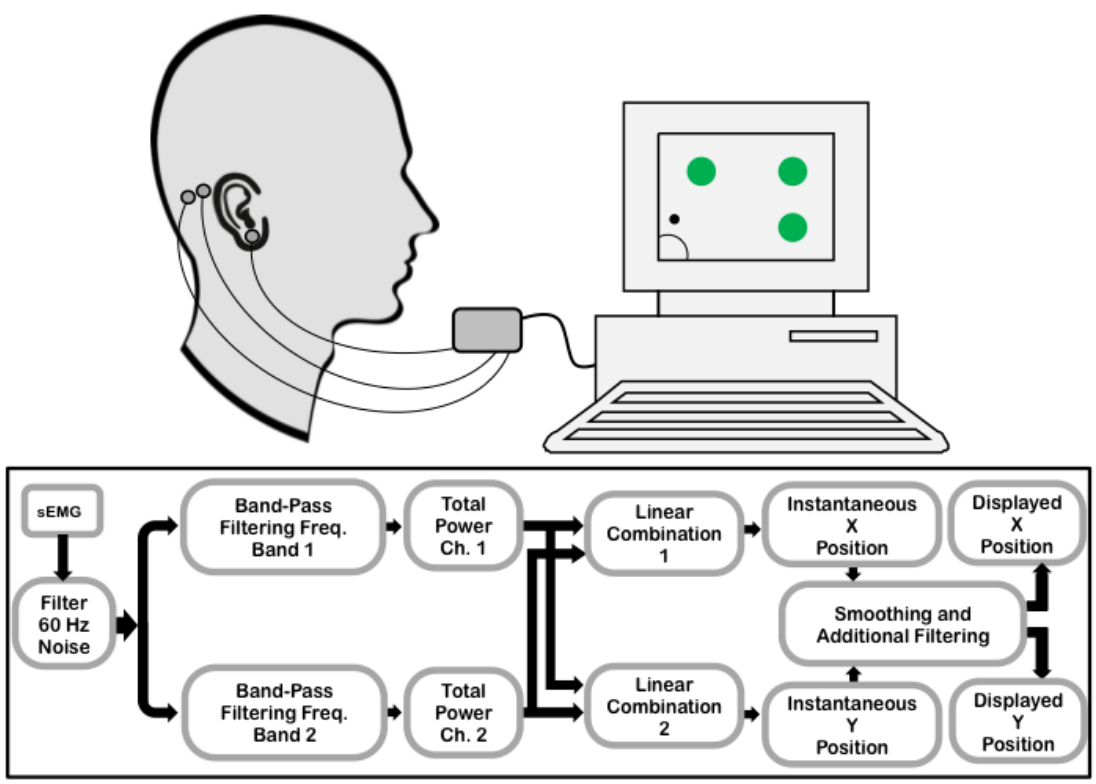

Figure 2. Differential pair of SEMG sensors are placed behind the ear above the AP muscle. A reference electrode is placed on the earlobe. Sensor wires connect to the amplifier and then to the computer. Computer screen shows the cursor, rest area (in the bottom left corner) and three targets. Below shows a flow chart of the DSP and filtering from the raw sEMG signal to the calculated cursor position. 
Before each session began, a calibration phase was completed in which the subjects maintained a maximum voluntary contraction (MVC) during a 5 second calibration period in order to compute MPB_1 and MPB_2, via computation of FFTs every 0.25 seconds. Two additional calibration coefficients $\left(E_{1}\right.$ and $E_{2}$ ) were used to scale the cursor position to ensure neither direction was biased for any given user, as well as to expand the space to allow the cursor to reach the $x$ and $y$-axes (since the SEMG signal can never have zero power in the bands). This scaling was done while the subject was moving the cursor around on the screen (no targets were present at this time). The coefficients ranged between 0 and 1 and were generally near 0.15 . Before the experiment began $E_{1}$ and $E_{2}$ were adjusted manually by the researcher to ensure the subject could reach all screen locations. Once $E_{1}, E_{2}, M P B \_1$ and MPB_2 were set, they could not be altered again during the sessions. Table 2 lists the final absolute powers in Band 1 and Band 2 required to hit each target after calibration.

Table 2. Final absolute powers required in Band 1 and Band 2 (required PowerB_1(t_final) and PowerB_2(t_final) in Equation 1) to hit each target after calibration. Computed by inverting Equation 1 using values of $X$ and $Y$ for each target and calibrated values of E1, E2, MPB_1 and MPB_2 for each subject. Units are $\left(\mathrm{mV}^{\wedge} 2\right)$. The large differences in the numerical values are the result of different muscles and different ability to access muscles for each subject. See Figure 3 for target locations on the screen.

\begin{tabular}{|c|c|cc|cc|cc|}
\hline \multirow{2}{*}{ Subject } & \multirow{2}{*}{ Session } & \multicolumn{2}{|c|}{ Target 1 } & \multicolumn{2}{c|}{ Target 2 } & \multicolumn{2}{c|}{ Target 3 } \\
& & Band 1 & Band 2 & Band 1 & Band 2 & Band 1 & Band 2 \\
\hline 1 & 1 & 547.04 & 315.38 & 1377.35 & 546.49 & 1289.85 & 413.55 \\
& 2 & 486.60 & 298.61 & 1196.05 & 496.18 & 1107.92 & 362.83 \\
\hline 2 & 1 & 668.67 & 628.03 & 1130.04 & 738.76 & 838.29 & 357.12 \\
\hline 3 & 1 & 590.27 & 525.75 & 1052.39 & 636.90 & 812.66 & 323.31 \\
\hline 4 & 1 & 126.75 & 50.50 & 359.47 & 118.54 & 352.59 & 107.59 \\
\hline 5 & 1 & 6.56 & 8.11 & 11.78 & 9.86 & 9.15 & 5.05 \\
\hline 6 & 1 & 121.00 & 132.30 & 263.20 & 187.20 & 229.80 & 117.18 \\
\hline 7 & 1 & 16.90 & 22.24 & 34.96 & 29.98 & 29.72 & 17.74 \\
\hline 8 & 1 & 10.18 & 10.41 & 20.54 & 13.74 & 17.20 & 7.91 \\
\hline
\end{tabular}

\subsection{EXPERIMENTAL PROTOCOL}

A custom graphical user interface was written in Matlab to show the full sEMG spectrum analysis in real time during the experiment. It also allowed for the recording of the full sEMG spectrum for each sample point. The subject was presented with one of three targets sized at $1 \%$ of the area of the screen on a separate monitor. Target 1 was placed at $25 \%$ of the screen area in the $x$-direction ( $X=25)$ and $75 \%$ of the screen area in the $y$-direction ( $Y=75$; relative to the bottom left corner of the screen). Similarly, Target 2 was placed at $75 \%$ of the screen area in both the $x$ - and $y$-directions, and Target 3 was placed at $75 \%$ of the screen area in the $x$-direction and $25 \%$ of the screen area in the $y$-direction (see Figure 3 ). 
In order for the cursor to hit the desired target, the subject was required to contract their muscle, which guided the cursor from the origin $(X=0, Y=0)$ to the target location. The power profiles were a function of each subject's calibration values (as described above) and therefore differed for each subject, and possibly from session to session (due to changes in E1 and E2), even though the target positions were fixed for everyone. It should be noted that subjects only needed a relatively small amount of normalized power (PowerB_1/MPB_1, PowerB_2/MPB_2) to hit even the far reaches of the screen; therefore subjects could also easily produce so much power as to take the cursor out of the field of view of the screen (which resulted in the cursor being pinned to the screen borders).

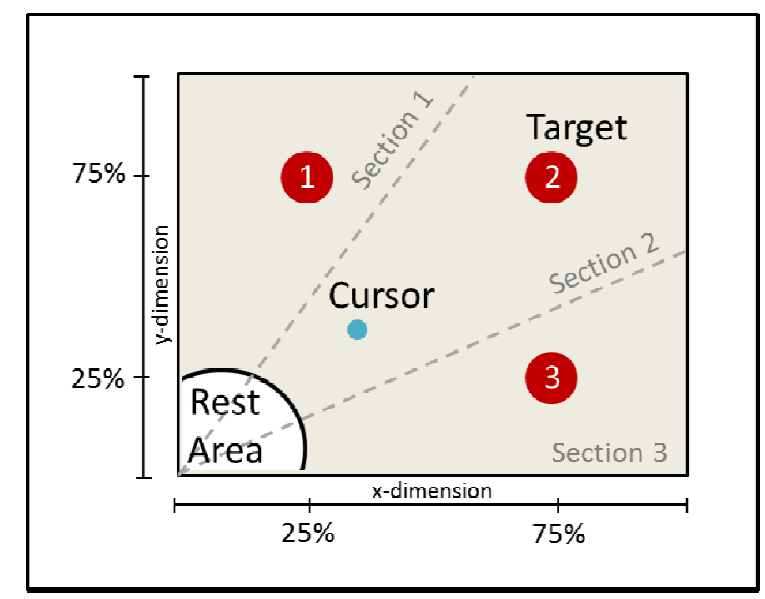

Figure 3. The location of the three targets on the screen (only one target was present at the screen at any time during the experiment, dotted lines denote screen areas used for analysis and are not shown to subjects).

The subject was presented with one target at a time in sequential order. Every three trials (in the order of Target 1 then 2 then 3) were referred to as a single 'cycle'. Each subject completed 100 cycles (300 trials) in a single session. For each trial, the cursor began in the bottom left corner in a rest area occupying $4 \%$ of the screen (Figure 3). As soon as the cursor exited the rest area, the subject was given 25 seconds to hit the target. When the target was hit, it turned green and the user was required to return the cursor to the rest area, via muscle relaxation, in order for the next target to appear. If the cursor did not hit the target in 25 seconds, the target disappeared and again the user was required to return the cursor to the rest area in order for the next target to appear. Thus, every trial was separated by a momentary muscle relaxation.

For Session 1, all eight subjects (four on EPL and four on AP) performed 300 trials without a break, unless necessary (only one AP subject took a single five minute break during one session). For Session 2, subjects 1-4 performed the same 300 trials; however after each 30-trial block, a static fatiguing exercise was performed to induce muscle fatigue. Only the EPL subjects took part in Session 2, as a comparable exercise to induce fatigue in the AP muscle was not possible. In all cases, the sessions lasted between 2.5 and 3 hours (including setup, calibration, and testing). In order to induce fatigue in the EPL muscle, an elastic band was placed around the hand as shown in Figure 1c. The subjects were asked to extend 
their thumb such that they felt muscle tension under the sensor site. They were instructed to maintain this static contraction until told to relax by the experimenter. During the contraction, the full frequency spectrum and median frequency (MDF) of the Power Spectral Density (PSD) were monitored by the experimenter to assess fatigue using standard measures. A well-established and often-used parameter to track muscle fatigue in static tasks is a downward shift in the MDF of the PSD (Basmajian, 1963; Gonzalez-Izal, et al., 2012; Mannion, et al., 1997; Merletti, et al., 1992; Tepavac \& Schwirtlich, 1997). We defined the muscle as sufficiently fatigued when the MDF dropped below $80 \%$ of the initial MDF recorded. Each fatiguing exercise lasted at least 90 seconds but was extended if a satisfactory level of fatigue had not yet been reached.

\section{RESULTS}

\subsection{SUCCESS RATES AND TIME-TO-TARGET}

We defined successful trials as all instances where the cursor hit or crossed over the target ${ }^{2}$. Correspondingly, we measured the time in seconds it took subjects to reach the target. Our analyses are concerned with any changes in performance (success rates and time-to-target) across use (trials). As fatigue may affect the power in the two separate frequency bands differently, we split the measures to investigate any differential performance on Target 1 and Target 3. This was because these two targets have non-overlapping screen co-ordinates. The data were analyzed using a Generalized Linear Model (GLM; identity link function; Gaussian variance function) relating the dependent variable (performance) to one quantitative independent variable (trials) and one categorical independent variable (target location).

Figure 4 shows the average success rates for each condition (AP and EPL Session 1 and 2) across trials (each data point represents a bin of ten trials) for Target 1 and Target 3. The side panel shows performance on target 1 and 3 on separate plots with individual subject data outlined in grey. For the AP subjects, there were no significant trends in success rates as a function of trials or target location, however for time-to-target there was a significant interaction between target location and trials $[\mathrm{t}(79)=$ 2.19, $p=0.032$ ]. This interaction seems to reflect that subjects were slower to reach Target 1 at the start of the session, whereas for most of the remaining trials they were slower to reach Target 3 . For the EPL subjects (Session 1), there were no significant effects of trials on performance, but success rates were significantly higher overall for Target 3 as opposed to Target $1[t(79)=2.77, p=0.007]$. There were no significant effects or interactions for time-to-target involving target location or trial.

During Session 2, when the EPL subjects were fatigued, there was an overall $26 \%$ decline in performance between the first and last bin of trials and this trend was significant $[t(79)=3.66, p<0.001]$. In addition, 
success rates were significantly higher overall for Target 3 compared to Target $1[t(79)=5.23, p<0.001]$ and finally, there was a significant interaction between trial and target location $[t(79)=2.00, p=0.05]$, confirming the pattern of steeper decline in success rates for Target 1 across the session. There was no significant change in time-to-target as a function of trials or target location.
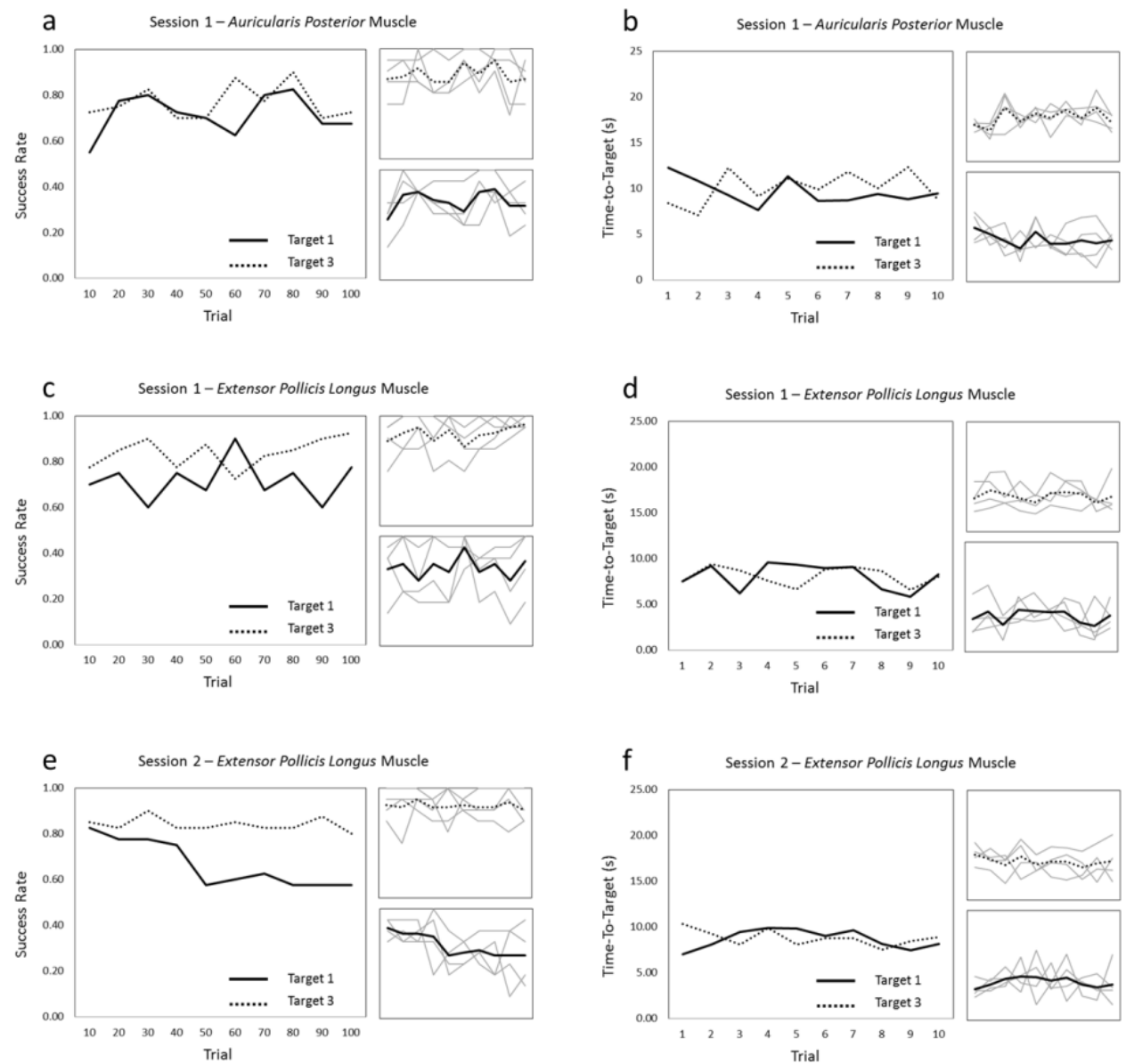

Figure 4 Performance (success rates and time-to-target) across trials for each condition a-b) AP Session 1, c-d) EPL Session 1 and e-f) EPL Session 2. Each of the main plots shows performance on Target 1 (solid line) and 3 (dotted line). The side panels of each plot show the performance on each target separately with individual subject data plotted in grey.

\subsection{CURSOR CONTROL}

To further investigate the potential consequences of fatigue, we looked at path based precision, as a measure of cursor control. For this analysis, the screen was divided into thirds of equal area with each target occupying one of the thirds as shown in Figure 3. For each trial, the percent of time spent in each third was calculated. As explained previously, each of the three targets was presented in a cycle, one after the other. In the current analysis, every three trials were averaged to a single data point (100 in total per subject), resulting in each point containing an attempt at all three target locations. We used this measure to investigate whether or not the cursor was biased towards any area of the screen - 
irrespective of which target the subjects intended to hit. As with our previous analyses we focus on the areas surrounding Target 1 and Target 3.

Figure 5 shows the time spent in each third of the screen averaged across subjects for each condition. In Session 2 (Figure $5 c$ ), the fatiguing exercises occurred after every ten data points (30 trials) and are indicated by vertical lines on the graph. For the purpose of the analysis we subtracted the time spent in Area 3 from the time spent in Area 1 to reveal any significant changes in the time the cursor spent in these areas of the screen across trials. We analyzed the data using a GLM with this difference score as the dependent variable and trials as the quantitative independent variable. The analyses confirmed that there were no changes in the difference score as a function of trials during Session 1 for the AP subjects. In contrast, there was a minor change in the difference score for the EPL subjects (7\%), which was marginally significant $[\mathrm{t}(398)=1.91, \mathrm{p}=0.06]$ and indicates that across trials subjects spent an increasing amount of time in Area 3 at the expense of Area 1. For the Session 2 data, the analysis revealed a 49\% change in the difference score as a function of trials and this trend was strongly significant $[\mathrm{t}(398)=$ $11.83, p<0.001$ ]. In addition to a very clear change in the percentage of time spent in Area 1 and 3 , from Figure 5 one can also observe very prominent spikes directly after each of the first four fatiguing exercises, and smaller, less defined 'bumps' following the rest of the fatiguing exercises. Each of these spikes shows that in most of the trials directly following fatiguing exercises, the cursor spends an increased amount of time in Area 3 irrespective of which target was attempted.
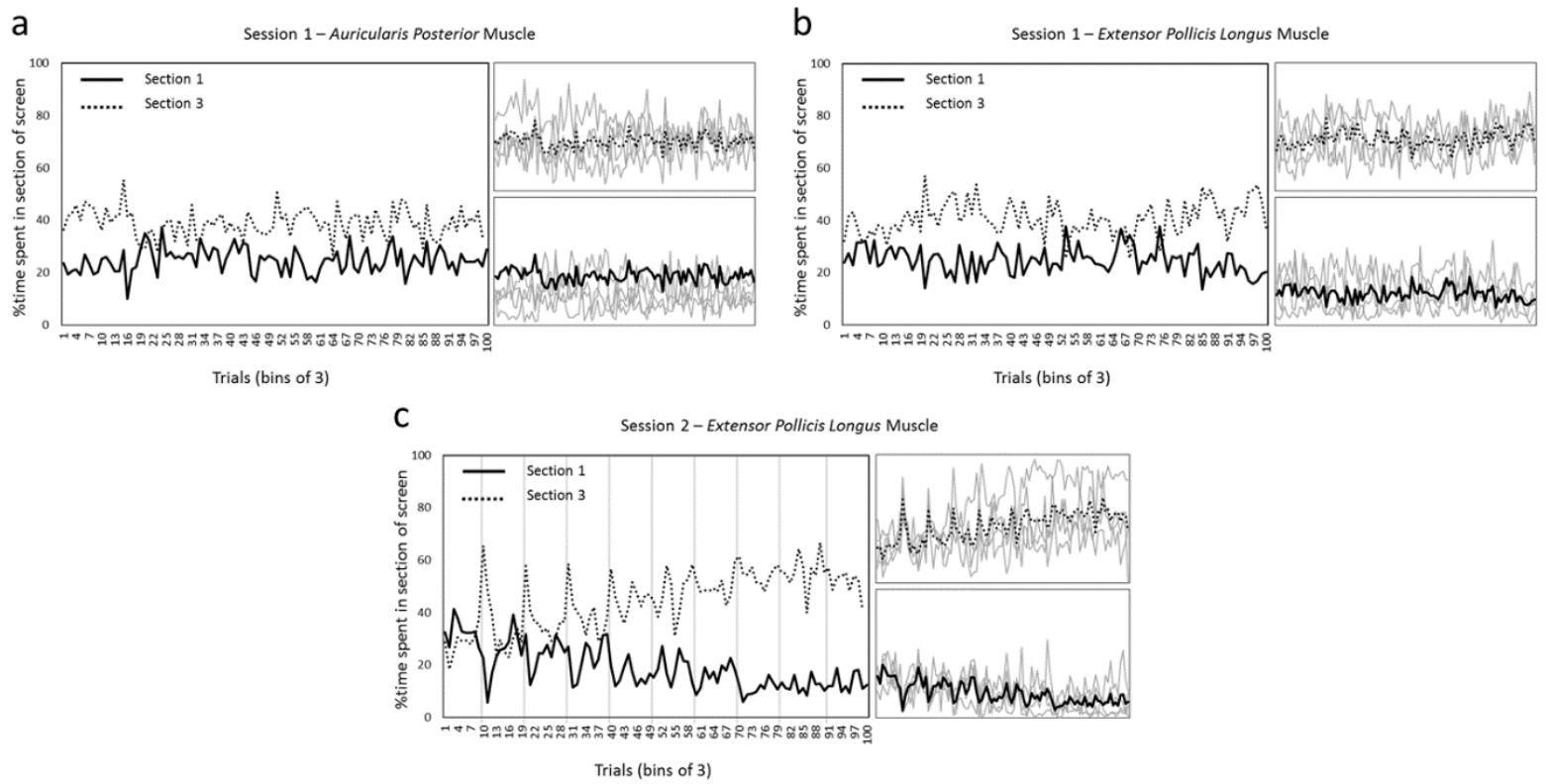

Figure 5. The lines represent the percentage of time spent in each third of the screen, averaged across subjects. The solid line represents the percent of time spent in Area 1 and the dotted line represents the percent of time spent in Area 3. a) Results from AP subjects during Session 1 (no fatiguing exercises) and b) Results from EPL subjects session during Session 1 (no fatiguing exercises) and c) Results from EPL subjects during Session 2 (with fatiguing exercises). The vertical grey lines indicate when a fatiguing exercise took place between trials. 


\subsection{SPECTRAL COMPRESSION DURING INTERSESSION FATIGUING EXERCISES}

It is generally accepted that as a muscle fatigues the Power Spectral Density (PSD) function of the sEMG signal during static isometric contractions experiences a spectral compression (Mannion, et al., 1997; Merletti, et al., 1992). This spectral compression is often described as a shift toward lower frequencies (Gonzalez-Izal, et al., 2012) and an increase in amplitude. In fact, a well-established and often-used parameter to track spectral compression and muscle fatigue, during static isometric contractions, is the median frequency (MDF) of the PSD (Basmajian, 1963; Gonzalez-Izal, et al., 2012; Mannion, et al., 1997; Merletti, et al., 1992; Tepavac \& Schwirtlich, 1997), defined as the frequency that divides the PSD into two regions of equal power (see Equation 2).

Equation 2.

$$
\int_{0}^{M D F} P(f) d f=\int_{M D F}^{\infty} P(f) d f
$$

We investigated spectral compression during the static contraction intersession fatiguing exercises described in Section 2.3. Averaged across all EPL subjects at the start of the initial static fatiguing exercise we found an MDF of $101 \mathrm{~Hz}$. At the end of the final fatiguing exercise (post block 9) the MDF was reduced to $70 \mathrm{~Hz}$. Additionally, at the end of the final fatiguing exercise, the power in Band 1 increased by $8 \%$ while the power value in Band 2 decreased by $26 \%$ (averaged across all EPL subjects) (see Figure 6).

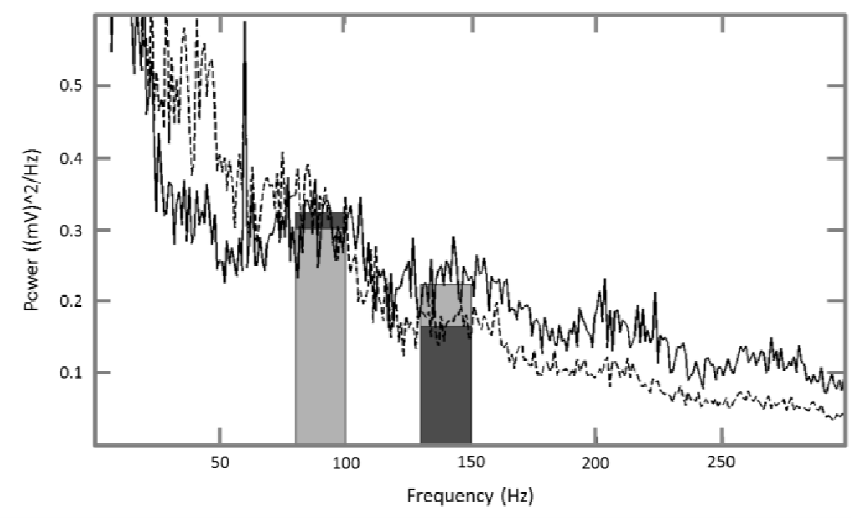

Figure 6. Average PSD across all EPL subjects for Session 2. Solid line represents the unfatigued PSD at the start of the initial static fatiguing exercise. Dashed line represents the fatigued PSD at the conclusion of the final static fatiguing exercise. Light grey bars show the power in each frequency band at the start of the initial static fatiguing exercise. Dark grey bars show the max power in each frequency band at the conclusion of the final static fatiguing exercise. 


\section{DISCUSSION}

The current study investigated the potential effects of muscular fatigue on the use of a myoelectric human-computer interface $(\mathrm{HCl})$. Eight subjects completed three-hour long cursor-to-target tasks using either the auricularis posterior (AP) or extensor pollicis longus (EPL). The EPL subjects took part in an additional session in which they completed a static fatiguing exercise between each block of 30 trials. When the subjects used the interface for extended time without intermittent fatiguing exercises, we found no clear evidence of fatigue. In Session 2, when fatiguing exercises were employed, the cursor overall spent more time in the bottom right area of the screen, suggesting difficulties moving in the $y$ direction. Correspondingly the success rates went down for Target 1, located at the top left area of the screen. We discuss our results below.

\subsection{SESSION 1}

For practical user interfaces, the muscle chosen will always be a product of his/her specific disability and preference. For Session 1, half the subjects used the AP muscle and the other half the EPL muscle. The AP muscle is a more likely candidate for use by subjects with paralysis as it is innervated at the brain stem rather than the spinal cord, making it accessible even by people with high spinal cord injury. We were concerned that the AP muscle, a less frequently used muscle, would fatigue faster than the EPL. We included both muscles to allow a comparison, and because the EPL muscle can be easily fatigued using an external exercise. The results from Session 1, revealed no effects on performance as function of use, with the exception of a significant interaction between target location and trials for time-to-target for the AP muscle. This interaction merely suggested that the time-to-target was higher for one target at the start of the session and higher for another towards the end. It is unclear what would cause such a pattern of results, however as no steady incline as a function of use was observed, we do not consider this interaction a likely indicator of fatigue. The only other significant result observed during Session 1 was an overall higher success rate for Target 3 compared to Target 1 for the EPL muscle. This target bias seemed to be present throughout the session (with an exception of one data point) and is therefore also an unlikely indicator of fatigue. Such a bias may have been caused by a slightly skewed calibration. Overall, we were surprised at the lack of any detrimental effects on performance as a function of use, suggesting that both muscles can be recruited for extended periods in a target-hitting paradigm.

All subjects in the current study had some experience with previous versions of our single site $\mathrm{HCl}$. We deliberately invited experienced subjects primarily to avoid any early learning confound in our performance data (previous studies have showed a steep learning curve during the initial few hours of interface training; see Skavhaug et al., 2016). One concern, however, is that the level of prior training protected the current subjects from physical fatigue that naive subjects would be more sensitive to. We cannot completely rule out this possibility; however for all our subjects (with the exception of one) several months had passed since the last performance session. Furthermore, the subjects' training time was limited to 2-8 hours, which seems unlikely to have affected their ability to withstand future fatigue. 
We believe that the primary reason why our device is relatively resistant to fatigue is the low level of contraction needed for control. Due to the limited literature on fatigue and SEMG-driven humancomputer interfaces, it is difficult to say whether devices that rely on total power from multiple sites would result in more or less fatigue, however this would be an interesting comparison to investigate in the future.

\subsection{SESSION 2}

Although performance did not decline with use for the EPL muscle during Session 1, there was a marginally significant trend for subjects to spend gradually less time in Area 1 (top left; surrounding Target 1) and more time in Area 3 (lower right; surrounding Target 3) of the screen. This trend may be an indicator of limited muscular fatigue, although the subjects were still able to successfully hit the same proportion of targets. Clear detrimental effects on performance were only observed in Session 2, when a fatiguing exercise was employed at every 30-trial interval. Fatigue appeared to cause a specific difficulty navigating the cursor in y-space, which likely caused the corresponding decline in success rates for the upper left target (Target 1). Directly following each of the first three fatiguing exercises, there were clear spikes in the percentage of time spent in Area 3 at the expense of Area 1, indicating that the subjects experienced brief phases during which they had even greater difficulties moving in the $y$ direction; however, they seemed to at least partially recover from this difficulty fairly quickly (see Figure 5). After the fourth fatiguing exercise the recovery appeared slower and less complete. Subsequently, the muscle did not recover and the cursor resided in Area 3 most of the time. The muscle fatigue and recovery patterns are consistent with the findings of Milner-Brown and Miller (1986) who, while investigating impaired impulse propagation during fatigue, showed that after one minute of static $100 \%$ maximum voluntary contraction (MVC), 45 to 240 seconds was required to recover back to normal values. After 2 minutes of $100 \%$ MVC it took from 2 to 10 minutes to recover.

Muscle fatigue is well known to change the spectral properties of SEMG signals, and presumably such changes account for the bias of cursor presence to Area 3 following fatigue. The $\mathrm{HCl}$ task involved a series of low-level dynamic contractions that collectively guided the cursor from the rest area to the target, meaning we were unable to analyze raw PSDs directly to observe spectral compression, as is common in static compression tasks ${ }^{3}$. However, some studies have hypothesized that spectral compression may also occur in dynamic tasks (see Arendt-Nielsen and Sinkjær, 1991). This may explain the pattern of cursor movements seen in session 2 of our study.

We hypothesize that the skill that each subject must master for our task is the creation of the proper amount of normalized power in each band, via proper muscle contractions. Normalized power encompasses the absolute power subjects voluntarily produce in each band divided by the maximum amount of power they are capable of producing (due to the physiological state of their muscles, see 
Equation 3). In our experiments, at the start of a session (pre-fatigue) the experimenter attempted to measure each subject's maximum power available in each band and encapsulate this into a value for MPB_1 and MPB_2 in Equation $1^{4}$. Once these values were set, MPB_1 and MPB_2 were fixed as constants throughout the experiment. As a result, the required absolute powers needed in each band were also fixed throughout sessions 1 and 2 (see Table 2 for required values). Importantly, later in the experiment, MPB_1 and MPB_2 may or may not represent each subject's actual maximum power available in each band as the muscle fatigues. In essence, the required PowerB_1 and PowerB_2 absolute power numerical values needed to hit each target did not change pre- and post-fatigue, but they represent very different muscle contractions (normalized powers). Since we cannot measure directly how the true maximum available powers change during our dynamic task, we used observed changes during the static intersession fatiguing exercise as a guide, and calculated normalized powers that the subjects needed to achieve after fatigue (averaged over all EPL subjects, see Equation 3 and Table 3). Recall after fatigue, in the intersession fatiguing exercise (static contraction), we found that the actual maximum power in Band 1 had increased by $8 \%$ while the maximum power value in Band 2 had decreased by $26 \%$ (averaged across all EPL subjects; see Figure 6). Fatigue dictates that subjects must learn new muscle contractions (muscle states) to hit the various target locations. The normalized powers values in Table 3 represent an average muscle state (contraction) that the subjects are required to achieve pre- and post-fatigue for the same target locations. Note that numerically, the muscle state needed to hit Target 3 after fatigue resembles the muscle state needed to hit Target 2 before fatigue (Table 3). However, the muscle states needed to hit Target 1 and Target 2 after fatigue are much different than for any of the targets pre-fatigue.

\section{Equation 3.}

For each target, where MPB_1 and MPB_2 were measured before fatigue and fixed:

$$
\text { Required Normalized Power Before Fatigue Band } 1 \text { = (Required PowerB_1)/MPB_1 }
$$

Required Normalized Power Before Fatigue Band 2 = (Required PowerB_2)/MPB_2

Required Normalized Power After Fatigue Band 1 = (Required PowerB_1)/(1.08*MPB_1)

Required Normalized Power After Fatigue Band 2 = (Required PowerB_2)/(.84*MPB_2) 
Table 3. Normalized power profiles needed to hit each target before and after fatigue (averaged over all EPL subjects). Required absolute powers required to hit each target (PowerB_1 and Power B_2) are constant before and after fatigue but the maximum power achievable (denominators in Equation 3) will change after fatigue.

\begin{tabular}{|c|c|c|c|c|}
\hline \multirow{2}{*}{ Target } & \multicolumn{2}{|c|}{ Before Fatigue } & \multicolumn{2}{c|}{ After Fatigue } \\
\cline { 2 - 5 } & Band 1 (x 100) & Band 2 (x 100) & Band 1 (x 100) & Band 2 (x 100) \\
\hline 1 & 8.011 & 10.558 & 7.417 & 14.268 \\
2 & 16.551 & 14.218 & 15.325 & 19.214 \\
3 & 14.057 & 8.399 & 13.016 & 11.351 \\
\hline
\end{tabular}

Instead of considering a constant target location and changing normalized powers for pre- and postfatigue, we can alternatively use Equation 1 and Table 3 to visualize a 'virtual target shift' when normalized powers are held constant. Note that the far right side vector of Equation 1 represents normalized powers in bands 1 and 2, corresponding to a particular muscle contraction. If we substitute the normalized powers for this vector before fatigue as in Table 3, we see these normalized powers map to our original desired target locations (see Figure 7). However, if we substitute the normalized powers for this vector after fatigue as in Table 3, we see that the target locations have 'shifted' (Figure 7). Put another way, if users had learned to create the needed normalized powers to hit the virtual targets before fatigue (given the maximum available powers before fatigue), they could have maintained the same normalized power profiles to hit the actual targets after fatigue (given the maximum available powers after fatigue). Of course, they did not practice hitting the virtual target locations before fatigue (only the real target locations), so the task after fatigue is presumably harder.

In the case of Targets 1 and 2, the virtual locations fall outside of the interface's computer screen altogether. As discussed in Section 2.3, only small amounts of power are required to pin the cursor to the sides of the computer screen. This means that following fatigue, subjects were required to produce entirely novel contractions. The subjects also had to learn a new normalized power profile to reach Target 3, but importantly, this normalized profile overlapped with the profile previously needed to hit Target 2 (in its original position). Hitting the 'virtual' Target 3 location therefore did not require the learning of a new normalized power profile, but rather involved associating an 'old' profile with a new location. In summary, the tendency for the cursor to spend more time in Area 3 of the screen at the expense of Area 1, may be indirect evidence of a spectral compression during the dynamic contraction task, but more direct investigations are needed before any definitive conclusions can be drawn. 


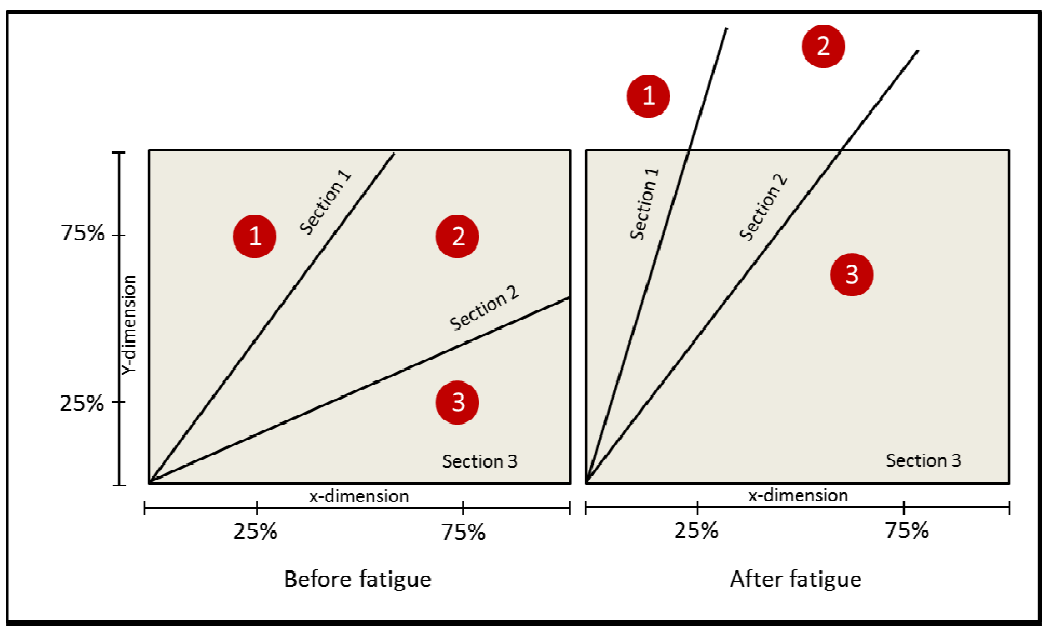

Figure 7. Following fatigue, the available maximum powers in the two frequency bands have changed. Consequently the normalized power profiles necessary for hitting the three targets also change. In practice, this can be compared to the experience of shifting target locations. Whereas target 3 has moved closer to target 2's original position, the two remaining targets have moved to an area outside the visible screen.

\subsection{PRACTICAL ISSUES AND LIMITATIONS}

The current study investigated the consequences of fatigue on the usability of our novel myoelectric $\mathrm{HCl}$, however there are many other factors besides fatigue that may influence the general usability of a device. When conducting long-duration experiments it is important to consider mental fatigue as well as physical fatigue. Our cursor-to-target paradigm is relatively attention demanding and subjects need to focus on the screen as they carry out the task. Continuous focus on a computer task for three hours is likely to cause some discomfort towards the end, however we did not monitor subjects' mental experience and are thus unable to estimate how likely this was to have affected the results. As our main fatigue related findings were restricted to session 2, we consider the gradual decline in cursor control to be primarily due to physical, as opposed to mental, fatigue. Challenges related to mental fatigue and the general attention demands of the $\mathrm{HCl}$ are interesting topics for future consideration. The current interface requires the user's full visual attention (this requirement might diminish with more extensive training), however it is designed for execution of discreet commands rather than for continuous use. Therefore, three hours of continuous use is highly unlikely and mental fatigue may not be likely to occur under more realistic circumstances.

Another factor that influences the usability of any device driven by bio-signals is the susceptibility to movement artifacts. Although SEMG signals have a better signal-to-noise ratio compared to electroencephalography (EEG) employed in brain-computer interfaces (e.g. Wolpaw and McFarland, 2004), our $\mathrm{HCl}$ requires the subject to sit relatively still during use. Subjects with immobility are most likely to use a head muscle such as the auricularis posterior for control, and activity recorded at this site can potentially be affected by activity from nearby muscles such as the larger temporalis, which is activated by biting down the jaw. We have, however, observed that several of our subjects in the 
current and past studies were able to speak and use the $\mathrm{HCl}$ simultaneously (unpublished). In addition, one pediatric subject with Tetra Amelia Syndrome (absence of all four limibs), who was tested in his home as part of a different study, frequently shifted his body, spoke, and tilted his head while using our device, all without causing major cursor movement problems.

The primary motivation behind our myoelectric $\mathrm{HCl}$ was to reduce the number of required electrode sites to only one, however a minimal recording configuration is associated with certain costs. For example, it can be argued that our device is lacking in intuitiveness as compared to devices that employ multiple recording channels (e.g. Cler \& Stepp, 2015; Williams \& Kirsch, 2008) which allow a direct mapping between one muscle and one movement direction. Although high intuitiveness may ensure a device is easier to use from the start, lower intuitiveness does not necessarily prevent a user from achieving high performance levels or high user satisfaction with sufficient training. For example, Radhakrishnan, Baker, \& Jackson (2008) directly compared learning of intuitive and non-intuitive algorithms for control of a cursor and found that although performance was superior for the intuitive algorithm at the start of the study, this advantage diminished steadily with practice. In prosthetics, Resnik, Klinger, Etter, \& Fantini (2014) showed that a non-intuitive foot-controlled upper arm prosthetic, which required weeks of learning, still produced high marks in user satisfaction. It is difficult to quantify the benefits of one $\mathrm{HCl}$ above another as this will depend on the user's situation. The five channel system introduced by Cler \& Stepp (2015) is unarguably more intuitive but also more intrusive as it requires the user to wear several electrodes on the face. Our one sensor channel can be easily concealed behind the user's ear. Furthermore, some subjects do not have multiple recording sites available and, for them, the minimal recording configuration is crucial. We believe that the exact advantages and disadvantages of all interfaces must be evaluated in light of the individual user's specific needs and preferences. Hence, a range of different devices are needed to serve the large and diverse community of disabled users.

\subsection{CONCLUSION}

We conclude that fatigue is not a major concern during typical use of our myoelectric human-computer interface, which requires low levels of muscle contraction and would normally be used over short periods of time. We were surprised, however, that our $\mathrm{HCl}$ performance was not hampered by long durations of testing, for either the AP or the EPL muscle sites, especially since the AP is not a commonly used muscle. Negative effects were observed only when fatigue was induced through external fatiguing exercises at regular intervals. This result bodes well for the use of head muscles for other EMG based human computer interfaces. In muscle physiology, spectral compression is a well-established consequence of fatigue during static isometric contractions, and has been suggested to occur also for dynamic contractions. Our experiments may provide indirect evidence of spectral compression during fatigue within a dynamic contraction task. In the future, other EMG-based human-computer interfaces could be used to further examine this topic. 


\section{ACKNOWLEDGMENTS}

We thank all our subjects for their time and effort. This research was supported by grants from The Hartwell Foundation and the National Science Foundation (grant number 0966963). We also thank Kenneth Lyons and Benjamin Vernon for helpful comments and suggestions to the current work. Finally, we take this opportunity to gratefully acknowledge the contributions of all the students and collaborating researchers that have contributed to the development and testing of previous incarnations of the interface described in this work. We particularly acknowledge the recent contributions of Benjamin Vernon, Rebecca Bobell, Britt Yazel, Jay Gokhale, Cindy Dao, David Randolph, Lauran Barry, Huanchun Chen, Shira Muroff, Anna Nemchuk, and Bryce Korte.

\section{FOOTNOTES}

1. This should not be confused for muscle contraction time. Individual cursor-to-target trials were limited to 25 seconds (in case no target was hit), but most trials concluded with a successful target hit in less time.

2. Each time the target was hit, the full spectrum data was recorded for later analysis. In order to record the spectrum at the time of striking the target it was imperative that the target strike coincided with the 0.25 second calculation of a cursor position. It is possible for the cursor position to be calculated such that it lies outside of the target and 0.25 seconds later a new cursor position is calculated on the opposite side of the target. In actual interface use, such an instant would be counted as a target hit, as the cursor passed through the target. As this would not provide a power spectrum tagged to a position on the target these trials were not presented to the subject as a proper hit. In order for a target hit to visually register on the screen (turn green from the subject's perspective), the position at the moment the cursor position was calculated needed to correspond to a location directly within the target area. This resulted in some trials where it appeared that the cursor had 'hit' the target several times, before it turned green. This was clearly explained to all subjects.

3. Generally, the MDF is not considered a reliable measure of fatigue during dynamic contractions. For example, during an intense running exercise up a 20\% grade, Ament, Verkerke, Bonga, and Hof (1996) produced a slight increase in MDF. During an extensive walking exercise, Arendt-Nielsen and Sinkjær (1991) found no change at all in MDF. They postulate that this is because two effects are competing during dynamic tasks; fatigue, resulting in MDF decrease and intramuscular temperature rise, resulting in MDF increase. 
4. The purpose of this calibration was to normalize the power in the bands (PowerB_1 and PowerB_2 in Equation 1) with the maximum available power values (MPB_1 and MPB_2 in Equation 1), and then to adjust the values in calibration coefficients $E_{1}$ and $E_{2}$ such that the subject could move the cursor equally to all regions of the screen. This procedure then dictated how much absolute power that subjects needed to produce in each band (PowerB_1 and PowerB_2 computed by inverting Equation 1 for each target location with known $E_{1}, E_{2}, M P B \_1$, and MPB_2) in order to reach the three target locations. 


\section{REFERENCES}

Ament, W., Verkerke, G. J., Bonga, G. J. J., \& Hof, A. L. (1996). Electromyogram median power frequency in dynamic exercise at medium exercise intensities. European Journal of Applied Physiology and Occupational Physiology, 74, 180-186.

Arendt-Nielsen, L., \& Sinkjær, T. (1991). Quantification of human dynamic muscle fatigue by electromyography and kinematic profiles. Journal of Electromyography \& Kinesiology, 1, 1-8.

Basmajian, J. (1963). Control and training of individual motor units. Science, 141, 440-441.

Chin, C. A., Barreto, A., Cremades, J. G., \& Adjouadi, M. (2008). Integrated electromyogram and eye-gaze tracking cursor control system for computer users with motor disabilities. Journal of Rehabilitation Research \& Development, 45, 161-174.

Choi, K., Sato, M., \& Koike, Y. (2006). A new, human-centered wheelchair system controlled by the EMG signal. Proceedings of the 2006 international joint conference on neural networks, 4664-4671.

Cler, M. J., \& Stepp, C. E. (2015). Discrete vs. Continuous Mapping of Facial Electromyography for Human-Machine-Interface Control: Performance and Training Effects. IEEE Transactions on Neural Systems and Rehabilitation Engineering, 23 (4), 1-9.

Engelhart, K., \& Hudgins, B. A. (2003). A Robust, Real-Time Control Scheme for Multifunction Myoelectric Control. IEEE Transactions on Biomedical Engineering, 40, 848-854.

Felzer, T., \& Freisleben, B. (2002). Hawcos: the hands-free wheelchair control system. Proceedings of the fifth international ACM conference on asistive technologies, 127-134.

Gonzalez-Izal, M., Malanda, A., Gorostiaga, E., \& Izquierdo, M. (2012). Electromyographic models to assess muscle fatigue. Journal of Electromyography and Kinesiology, 22, 501-512.

Hands, G. L., \& Stepp, C. E. (2016). Effect of Age on Human-Computer Interface Control Via Neck Electromyography. Interacting with Computers, 28 (1), 47-54.

Jiang, N., Rehbaum, H., Vujaklija, I., Graimann, B., \& Farina, D. (2013). Intuitive, online, simultaneous and proportional myoelectric control over two degrees of freedom in upper limb amputees. IEEE Transaction on Neural Systems and Rehabilitation Engineering, 22, 501 - 510.

Lyons, K. R., \& Joshi, S. S. (2013). Paralyzed Subject Controls Telepresence Mobile Robot using Novel sEMG Brain-Computer Interface: Case Study. Proceedings of the IEEE International Conference for Rehabilitation Robotics, 1-6. 
Mannion, A. F., Connolly, B., Wood, K., \& Dolan, P. (1997). The use of surface EMG power spectral analysis in the evaluation of back muscle function. Journal of Rehabilitation Research \& Development, 34, 427-439.

Mello, R. G., Oliveira, L. F., \& Nadal, J. (2007). Digital butterworth filter for subtracting noise from low magnitude surface electromyogram. Computer Methods and Programs in Biomedicine, 87, 2835.

Merletti, R., Knaflitz, M., \& DeLuca, C. J. (1992). Electrically evoked myoelectric signals. Critical Reviews in Biomedical Engineering, 19, 293-340.

Milner-Brown, H. S., \& Miller, R. G. (1986). Muscle membrane excitation and impulse propagation velocity are reduced during muscle fatigue. Muscle and Nerve, 9, 367-374.

Moon, I., Lee, M., Chu, J., \& Mun, M. (2005). Wearable EMG-based HCl for electric-powered wheelchair users with motor disabilities. Proceedings of the 2005 IEEE Internatinoal Conference on Robotics and Automation, 2649-2654.

Nguyen, K., \& Nguyen, T. (2011). Using electrooculogram and electromyogram for powered wheelchair. Proceedings of the 2011 IEEE International Conference on Robotics and Biomimetics, 1585-1590.

Oonishi, Y., Oh, S., \& Hori, Y. (2008). New control method for power assisted wheelchair based on upper extremity movement using surface myoelectric signal. 10th IEEE International workshop on advcanced motion control, 498-503.

Oskoei, M. A., \& Hu, H. (2007). Myoelectric Control Systems-A survey. Biomedical Signal Processing and Control, 2, 275-294.

Park, E., \& Meek, S. (1993). Fatigue compensation of the electromyographic signal for prosthetic control and force estimation. IEEE Transactions on Biomedical Engineering, 40, 1019-1023.

Perez-Maldonado, C., Wexler, A. S., \& Joshi, S. S. (2010). Two-dimensional Cursor-to-target Control from Single Muscle Site sEMG Signals. IEEE Transactions on Neural Systems and Rehabilitation Engineering, 18, 203-209.

Radhakrishnan, S. M., Baker, S. N., \& Jackson, A. (2008). Learning a novel myoelectric-controlled interface task. Journal of neurophysiology, 100, 2397-2408.

Resnik, L., klinger, S. L., Etter, K., \& Fantini, C. (2014). Controlling a multi-degree of freedom upper limb prosthesis using foot controls: user experience. Disability and Rehabilitation: Assistive Technology, 9, 318-329.

Roche, A. D., Rehbaum, H., Farina, D., \& Aszmann, O. C. (2014). Prosthetic Myoelectric Control Strategies: A Clinical Perspective. Current Surgery Reports, 2. 
Scheme, E., \& Englehart, K. (2011). Electromyogram pattern recognition for control of powered upperlimb prostheses: state of the art and challenges for clinical use. Journal of Rehabilitation Research \& Development, 48, 643-660.

Skavhaug, I., Bobell, R., Vernon, B., \& Joshi, S.S. (2012). Pilot study for a brain-muscle-computer interface using the extensor pollicis longus with preselected frequency bands. Proceedings of the IEEE Conference in Engineering of Medicine and Biology Society, 1727-1731.

Skavhaug, I., Lyons, K., Nemchuk, A., Muroff, S., \& Joshi, S. S. (2016). Learning to modulate the partial powers of a single sEMG power spectrum through a novel human-computer interface. Human Movement Science, 47, 60-69.

Song, J., Jung, J., Lee, S., \& Bien, Z. (2009). Robust EMG pattern recognition to muscular fatigue effect for powered wheelchair control. Journal of Intelligent \& Fuzzy Systems, 20, 3-12.

Tepavac, D., \& Schwirtlich, L. (1997). Detection and prediction of fes-induced fatigue. Journal of Electromyography \& Kinesiology, 7, 39-50.

Thorp, E. B., Larson, E., \& Stepp, C. E. (2014). Combined Auditory and Vibrotactile Feedback for HumanMachine-Interface Control. IEEE Transactions on Neural Systems and Rehabilitation Engineering, $22(1), 62-68$.

Vernon, S., \& Joshi, S. S. (2011). Brain-Muscle-Computer Interface: Mobile phoneprototype development. IEEE Transactions on Information Technology in Biomedicine, 15, 531-538.

Wei, W., Hu, H., \& Yuan, K. (2009). Use of forehead bio-signals for controlling an intelligent wheelchair. Proceedings of the 2008 IEEE international conference on robotics and biomimetics, 208-113.

Weisz, J., Barszap, A. G., Joshi, S. S., \& Allen, P. K. (2014). Single Muscle Site sEMG Interface for Assistive Grasping. Conference Proceedings of the IEEE International Conference on Intelligent Robots and Systems, 7.

Williams, M. R., \& Kirsch, R. F. (2008). Evaluation of head orientation and neck muscle EMG signals as command inputs to a human-computer interface for individuals with high tetraplegia. IEEE Transactions on Neural Systems and Rehabilitation Engineering, 16, 485-496.

Wolpaw, J. R., \& McFarland, D. J. (2004). Control of a Two-Dimensional Movement Signal by a Noninvasive Brain-Computer Interface in Humans. Proceedings of the National Academy of Sciences of the United States of America, 101, 17849-17854.

Young, A. J., Smith, L. H., Rouse, E. J., \& Hargrove, L. J. (2014). A comparison of the real-time controllability of pattern recognition to conventional myoelectric control for discrete and simultaneous movements. Journal of Neural Engineering and Rehabilitation, 11, 34-44. 
Zecca, M., Micera, S., Carrozza, M. C., \& Dario, P. (2002). Control of Multifunctional Prosthetic Hand by Processing the Electromyographical Signal. Critical Reviews in Biomedical Engineering, 30, 459485. 